\title{
CORRIGENDUM TO "PROTOTYPE OF WEB-BASED DAILY WORK REPORT MANAGEMENT SYSTEM USING SMART PENS"
}

Prof. Dr. Gradimir Danon, Editor in Chief of the Journal of Applied Engineering Science demanded the change in the list of the contributing authors.

\section{CLARIFICATION}

Upon written remarks of Dr. Alexander Babanin from University of Melbourne, Melbourne, Australia and Dr. Bertrand Chapron from Laboratoire d'Océanographie Physique et Spatiale, Centre de Brest, IFREMER, Plouzané, France that each of them was included in the list of co-authors without their acceptance and knowledge, and written apology of the corresponding author Dr. John Chen explaining it was his omission, the Editorial Board decided to correct the list of initially listed authors.

Therefore, initial authors list from the above stated paper published under the paper number 17(2019)3, 607, 280283 with DOI:10.5937/jeas10-18602:

Tim Chen ${ }^{1}$, Alexander Babanin², Abū al-Qāsim Muhammad³, Bertrand Chapron", John Chen ${ }^{5,6 *}$, Himel Md Sakibul Hassan Sajib6,7

${ }^{1}$ AI LAB, Faculty of Information Technology, Ton Duc Thang University, Ho Chi Minh City, Vietnam

${ }^{2}$ University of Melbourne, Melbourne, Australia

${ }^{3}$ King Abdulaziz University, Jeddah, Saudi Arabia

${ }^{4}$ Laboratoire d'Océanographie Physique et Spatiale, Centre de Brest, IFREMER, Plouzané, France

${ }^{5}$ Staffordshire University, Staffordshire, United Kingdom

${ }^{6}$ BRAC University, Dhaka, Bangladesh

${ }^{7}$ Asia Pacifi c University, Kuala Lumpur, Malaysia

has to be written as follows:

Tim Chen ${ }^{1}$, Abū al-Qāsim Muhammad', John Chen ${ }^{3,4 *}$, Himel Md Sakibul Hassan Sajib ${ }^{4,5}$

${ }^{1} A$ I LAB, Faculty of Information Technology, Ton Duc Thang University, Ho Chi Minh City, Vietnam

${ }^{2}$ King Abdulaziz University, Jeddah, Saudi Arabia

${ }^{3}$ Staffordshire University, Staffordshire, United Kingdom

${ }^{4}$ BRAC University, Dhaka, Bangladesh

${ }^{5}$ Asia Pacific University Kuala Lumpur, Malaysia

Exclusion of Dr. Alexander Babanin and Dr. Bertrand Chapron does not affect original paper. 